\title{
Rischio di mortalità con i farmaci antipsicotici nella popolazione psichiatrica anziana? Una revisione sistematica della letteratura
}

\section{Mortality risks with antipsychotic drugs in a population of elderly psychiatric persons? A systematic review of the literature}

\author{
Riccardo De Giorgi ${ }^{*}$, Andrea Cipriani ${ }^{* *}$
}

Riassunto: in Italia gli anziani raggiungono quasi un quarto della popolazione generale. In questo sottogruppo, gli antipsicotici sono ampiamente prescritti: nonostante siano autorizzati esclusivamente per schizofrenia e disturbo bipolare, è assai frequente l'uso off-label in demenza, delirium, ed altri disturbi. Un'ampia letteratura ha osservato un'associazione tra rischio di mortalità ed esposizione ad antipsicotici nella popolazione anziana con demenza, supportando la pubblicazione di black box warnings da parte della Food and Drug Administration negli Stati Uniti, secondo un percorso poi seguito dagli altri Paesi. Tuttavia, non è chiaro se tale associazione sia causale, così come se sia applicabile anche a pazienti anziani non affetti da demenza. Questo studio si pone l'obiettivo di revisionare sistematicamente la letteratura riguardante il rischio di mortalità nella popolazione geriatrica affetta da psicosi dello spettro schizofrenico e bipolare, demenza e delirium. È stata condotta una ricerca sistematica sui database MEDLINE e Cochrane Library, includendo quegli studi riguardanti la valutazione del rischio di mortalità in soggetti di 65 o più anni trattati con antipsicotici di prima elo seconda generazione. In totale, 25 pubblicazioni soddisfacevano i criteri d'inclusione per questa revisione sistematica: 3 per psicosi in schizofrenia e disturbo bipolare, 20 per demenze, e 2 per delirium. Sebbene non sia possibile rifiutare l'ipotesi di un soggiacente meccanismo fisiopatologico comune alla condizione "vecchiaia", comportante un maggiore rischio di mortalità a seguito dell'esposizione a farmaci antipsicotici, è innegabile che anche la patologia neuropsichiatrica di base abbia una sua influenza, sia essa positiva o negativa, su tale rapporto. La corretta valutazione dei fattori di rischio individuali può aiutare a prendere decisioni condivise tra clinico e paziente verso scelte terapeutiche più efficaci, sicure, e soprattutto più appropriate.

Parole Chiave: antipsicotici, mortalità, anziani, psicogeriatria, revisione sistematica.

*MD, Oxford Health NHS Foundation Trust, Oxford, United Kingdom.

${ }^{* *}$ MD PhD, Oxford Health NHS Foundation Trust, Oxford, United Kingdom, Department of Psychiatry, University of Oxford, Oxford, United Kingdom. 
Abstract: nowadays, in Italy, elderly people represent almost a quarter of the population. Antipsychotics, in this age group, are widely prescribed. Albeit these medications are approved specifically for schizophrenia and bipolar disorders, yet offlabel use of antipsychotics - in dementia, delirium, and other disorders - is recurrent. Literature has extensively highlighted the association between mortality risks and exposure to antipsychotics in elderly people with dementia - as underlined by the publication of the black box warnings from the US Food and Drug Administrationand subsequently taken into account by many other countries. Nonetheless, it remains unclear whether this association is causal, as well as whether it should be applied to elderly patients not suffering from dementia. This study presents a systematic review of the literature (searching MEDLINE and Cochrane Library databases) on the risks of mortality in a geriatric population suffering from psychosis of the schizophrenic and bipolar spectrum, dementia and delirium. The review includes the studies assessing the risk of mortality in elderly persons, aged 65 and over, treated with first and/or second generation antipsychotics. Overall, 25 publications met the inclusion criteria for this systematic review: 3 for psychosis in schizophrenia and bipolar disorder, 20 for dementia and 2 for delirium. Even if the hypothesis of a basic physiopathological mechanism associated with "old age" and involving a greater risk of mortality with the exposure to antipsychotic drugs cannot be discarded, nonetheless the underlying neuropsychiatric disease undeniably has also a specific, positive or negative, influence on this relationship. An accurate assessment of individual risk factors can help clinicians and patients in taking shared decisions on effective, safe, and above all appropriate therapeutic options.

Key Words: antipsychotics, mortality, elderly, psycho-geriatrics, systematic review.

\section{Scopo dello studio}

Nei Paesi Industrializzati la riduzione del tasso di natalità ed il contemporaneo aumento dell'aspettativa di vita spiegano il progressivo invecchiamento della popolazione. In Italia, la popolazione anziana (65 anni e oltre) raggiunge il $21.7 \%$, includendo un incremento nel numero dei "grandi anziani" (80 anni e oltre), che incidono per il 6.5\% degli abitanti [1].

In letteratura, è noto come i pazienti affetti da disturbi psichiatrici siano esposti ad un significativo incremento del rischio di mortalità con un pooled risk ratio (RR) di 2.22 (95\% Confidence Interval (CI), 2.12-2.33) [2], ed una riduzione dell'aspettativa di vita pari a 10-20 anni rispetto alla popolazione generale [3]. Cause non-naturali (suicidio) [4], ma soprattutto naturali (malattie fisiche) [5], in un contesto spesso caratterizzato da uno stile di 
vita caotico ed imprudente, contribuirebbero in rapporto sinergico negativo all'eccesso di mortalità per questi pazienti [6].

Tra gli psicofarmaci, gli antipsicotici sono i più significativamente associati a patologie cardiovascolari, cerebrovascolari, respiratorie, gastrointestinali, renali, endocrino-metaboliche (obesità, diabete mellito, dislipidemie, tiroidopatie, iponatremia) ed ematologiche, con un rischio relativo maggiore negli anziani per ognuna di queste condizioni [7]. Inoltre, gli antipsicotici sia di prima generazione che di seconda generazione sono stati associati ad un maggior rischio di morte cardiaca improvvisa, con adjusted incidencerate ratio (IRR) di 1.99 (95\% CI, 1.68-2.34) e 2.26 (95\% CI, 1.88-2.72), rispettivamente [8]; nello specifico sottogruppo degli ultrasessantacinquenni, antipsicotici noti per rischio di torsade de pointes presentano un odds ratio (OR) pari a 4.57 (95\% CI, 4.37-4.78) di mortalità, mentre antipsicotici conosciuti per potenziale rischio hanno un OR di 2.58 (95\% CI, 2.52-2.64), ed antipsicotici non associati al rischio di torsione di punte esibiscono comunque un OR di 2.14 (95\% CI, 2.03-2.65) [9]. In pratica, con l'avanzare degli anni, la popolazione geriatrica sarebbe maggiormente esposta al rischio di sviluppare eventi avversi da farmaci antipsicotici [10], pur non mancando studi che riportano evidenze opposte [11].

A fronte di ciò, gli psicofarmaci sono ampiamente prescritti nella popolazione anziana ([12],[13]) e, fra questi, l'utilizzo di antipsicotici è sproporzionatamente maggiore [14]; in un recente lavoro è stato osservato che, al momento dell'ospedalizzazione, il semplice fatto di avere più di 75 anni esponga ad un incremento del rischio di essere iniziato ad un antipsicotico (RR $=1.4,95 \%$ CI, $1.2-1.7$ ) [15]. In Italia, circa il 3\% dei pazienti anziani residenti in comunità è trattato con farmaci antipsicotici [16]. In realtà, la prescrizione di farmaci antipsicotici negli anziani è autorizzata esclusivamente per le psicosi dello spettro schizofrenico e bipolare; tuttavia, è assai frequente l'uso off-label per il trattamento di sintomi cognitivo-comportamentali correlati a disturbi di natura organica, quali la demenza, il delirium, ed altre ancora [17]. In questo contesto, 1'Agenzia Italiana del Farmaco autorizza l'utilizzo del solo risperidone

“(...) per il trattamento a breve termine (meno di 6 settimane) dell'aggressività persistente in pazienti con demenza di Alzheimer di grado da moderato a grave, che non rispondono ad approcci non farmacologici, e quando esiste un rischio di nuocere a se stessi o agli altri" [18].

Se i risultati di uno studio hanno dimostrato che circa la metà degli antipsicotici atipici veniva prescritto off-label, nonostante nella maggior parte dei casi fossero carenti i dati a supporto di tale pratica [19], nei pazienti più 
vecchi gli antipsicotici sono quasi esclusivamente usati per indicazioni offlabel [20]. Pertanto, terapie off-label sono adottate con rilevante frequenza e con un ottimismo terapeutico non sempre appropriato, nonostante vi sia un'allarmante insufficienza di trial clinici nella popolazione anziana, dato del tutto prevedibile se si tiene conto che i committenti, quasi sempre aziende farmaceutiche, tendono a non includere gli anziani nei trial e neppure essere interessati a sviluppare studi in questa popolazione, considerate le numerose situazioni fortemente predittive di insuccesso: le frequenti comorbidità mediche, il comune utilizzo di politerapie farmacologiche, i possibili deficit cognitivi, l'incrementata probabilità di sviluppare effetti collaterali e quindi, in definitiva, il maggior rischio di mortalità [21].

Storicamente, gli antipsicotici atipici sono stati largamente utilizzati nei pazienti anziani, a fronte di una teorica, migliore tollerabilità associata ad una minore probabilità di causare sintomi extrapiramidali rispetto agli antipsicotici di prima generazione [22]. In particolare, destavano preoccupazione le potenziali alterazioni farmacocinetiche e farmacodinamiche età-correlate con conseguente aumento del rischio di effetti collaterali motori, di sedazione e di cadute associate agli antipsicotici convenzionali [23]. Inoltre, era già stata documentata una minore incidenza di discinesie tardive nella popolazione geriatrica con l'utilizzo di antipsicotici di seconda generazione rispetto a quelli precedenti [24]. Sebbene l'associazione tra antipsicotici ed un profilo metabolico sfavorevole, con insorgenza di obesità, dislipidemie e diabete di tipo 2, iniziasse ad emergere nei pazienti più giovani [25], non vi erano studi in grado di confermare tali rischi all'interno della popolazione anziana [26]. In questo contesto, si inquadrava un ampio studio su 139.714 anziani istituzionalizzati in case di cura statunitensi negli anni 1999-2000, in cui 86.514 residenti presentavano dei disturbi cognitivo-comportamentali e, di questi, il $18.2 \%$ aveva ricevuto un trattamento antipsicotico, di cui $11 \%$ con antipsicotici di seconda generazione e $6.8 \%$ con antipsicotici di prima generazione [27]. Tuttavia nel 2004, la Committee on Safety of Medicines riportava un allarmante incremento di eventi cerebrovascolari nella popolazione geriatrica affetta da demenza e sottoposta a trattamento con antipsicotici atipici [28]. Un anno più tardi, la Food and Drug Administration (FDA), basandosi largamente su una precedente meta-analisi di 15 studi randomizzati controllati la quale aveva osservato un pooled OR di 1.54 (95\% CI, 1.06-2.23) tra utilizzo di antipsicotici di seconda generazione in anziani con demenza e mortalità [29], pubblicava un primo black box warning, che riportava un aumento della mortalità di circa 1.6-1.7 volte in soggetti anziani con demenza trattati con antipsicotici di seconda generazione [30]. Tuttavia, gli stessi autori della meta-analisi richiedevano cautela nell'interpretazione di questi dati, in quanto non era stato possibile individuare alcuna causa 
specifica in grado di spiegare tale incremento di mortalità, giacché gran parte dei decessi osservati aveva origine cardiaca (scompenso cardiaco, morte cardiaca improvvisa) o respiratoria (polmonite), cioè quelle cause ordinariamente associate a morte nelle demenze [29]. Nuovamente nel 2008 la FDA, sulla base di tre studi retrospettivi che avevano osservato che gli antipsicotici di prima generazione determinavano rischi anche maggiori rispetto ai corrispettivi di nuova generazione, con $\mathrm{RR}=1.4$ (95\% CI, 1.31.5) ([31]-[33]), estendeva la precedente avvertenza anche agli antipsicotici convenzionali [34].

È importante notare come gran parte delle questioni oggetto di studio abbiano interessato un sottogruppo della popolazione anziana, nello specifico quello affetto da demenze; su questa linea, si è pertanto sviluppata negli anni una vasta letteratura, per lo più osservazionale, i cui risultati tuttavia sembrano ancora lontani dall'essere considerati inequivocabili e definitivi. La situazione è perlomeno altrettanto critica ove si consideri l'utilizzo di antipsicotici in una popolazione anziana non affetta da demenza: tale preoccupazione è plausibile giacché non si può escludere che, alla base del potenziale incremento di mortalità osservato dalla FDA nei pazienti anziani con demenza a seguito dell'utilizzo di antipsicotici, vi sia un meccanismo fisiopatologico correlato non solo con la condizione "demenza", ma anche ed ancor più in generale con la "senilità" ed il processo di invecchiamento [35]. In altre parole, qual è il rischio, in termini di incremento di mortalità, a cui sottoponiamo i pazienti psichiatrici anziani nel momento in cui prescriviamo un farmaco antipsicotico?

Questo studio si propone l'obiettivo di fornire una risposta a tale domanda, attraverso una revisione sistematica della letteratura riguardante l'associazione tra il rischio di mortalità e la prescrizione di antipsicotici all'interno della popolazione geriatrica (65 anni e oltre) affetta da schizofrenia, disturbo bipolare, demenza e delirium.

\section{Metodi}

Come prima cosa sono stati individuati i termini MeSH pertinenti: "aged" o "aged, 80 and over", "antipsychotic agents" e "mortality". Tali termini, insieme con i lemmi generici "elderly", "older adults", "antipsychotics" e "death" per tutti i campi, sono stati impiegati nell'algoritmo di ricerca sui database MEDLINE e Cochrane Library. In tal modo, sono stati inclusi quegli studi riguardanti la valutazione del rischio di mortalità in soggetti di 65 o più anni trattati con antipsicotici di prima e/o seconda generazione. La search è stata finalizzata nel marzo 2016, includendo revisioni sistematiche 
(con o senza meta-analisi) e studi osservazionali di coorte e caso-controllo pubblicati sino a tale data, in lingua inglese o italiana. Ulteriori pubblicazioni significative sono state aggiunte a seguito di una hand-search della bibliografia degli articoli precedentemente individuati; gli articoli ripetuti nella ricerca iniziale della letteratura sono stati eliminati.

Qualora un articolo non fosse specifico per pazienti anziani, ma analizzasse una popolazione di età mista, ne sono state estrapolate le informazioni riguardanti la fetta di popolazione oltre i 65 anni. Ogniqualvolta questo non sia stato possibile, perché i dati del campione non erano stati analizzati separatamente per fasce di età, o perché nella fetta di popolazione più anziana venivano inclusi pazienti molto al di sotto dei 65 anni, lo studio è stato scartato.

Inoltre, la popolazione oggetto di indagine è stata selezionata per includere i soli studi su pazienti psichiatrici afferenti alle tre maggiori sfere di utilizzo, autorizzate od off-label, dei farmaci antipsicotici nell'età anziana, cioè psicosi in schizofrenia e disturbo bipolare, demenze, e delirium; pertanto, sono stati esclusi dai risultati quei lavori che riportavano dati di mortalità associata ad antipsicotici in una popolazione geriatrica generale non meglio specificata, accogliendo quindi anche pazienti non-psichiatrici, pur se tali articoli sono stati considerati in fase di discussione.

Infine, studi volti alla comparazione, in termini di mortalità, tra antipsicotici di prima generazione versus antipsicotici di seconda generazione, senza alcun confronto con pazienti non in trattamento, sono stati rimossi.

Seguendo la metodologia standard delle revisioni sistematiche, i titoli ed abstract degli articoli trovati sono stati esaminati per l'inclusione (RDG), qualora rispondessero ai criteri sopra riportati; in caso di dubbi sulla attinenza e/o pertinenza con la fattispecie di cui ci occupiamo, l'articolo è stato letto per intero e valutato da un secondo ricercatore (AC).

I risultati sono stati suddivisi e discussi in tre grandi aree tematiche, nel cui contesto questi farmaci vengono principalmente utilizzati nell'anziano: psicosi in schizofrenia e disturbo bipolare, demenze, e delirium. Per ognuna di queste aree, le referenze sono state suddivise, per tipologia di indagine e secondo la gerarchia delle evidenze, in revisioni sistematiche/meta-analisi e studi osservazionali.

\section{Risultati}

Attraverso l'algoritmo di ricerca esposto, si sono ottenuti inizialmente 380 record dal database MEDLINE e 48 dalla Cochrane Library, cui ne sono stati aggiunti altri 9 acquisiti tramite hand-search della bibliografia. Dopo aver eliminato 5 articoli duplicati, in totale, 432 record sono stati 
sottoposti a screening per titolo ed abstract, secondo i criteri d'inclusione ed esclusione sopra riportati. Da questa prima selezione, 56 articoli sono stati quindi valutati full-text, conducendo ad escluderne altri 31. Il numero finale è di 25 pubblicazioni incluse nella revisione sistematica: 3 per psicosi in schizofrenia e disturbo bipolare (nessuna revisione sistematica/meta-analisi e 3 studi osservazionali, di cui 1 ad includere anche disturbo bipolare), 20 per demenze (6 revisioni sistematiche/meta-analisi e 17 studi osservazionali), e 2 per delirium (1 revisione sistematica/meta-analisi e 1 studio osservazionale).

I risultati dei diversi studi sono stati resi principalmente in termini di risk ratio/relative risk ( $\mathrm{RR})$, risk difference ( $\mathrm{RD} \%)$, hazard ratio $(\mathrm{HR})$, odds ratio (OR), e number needed to harm $(\mathrm{NNH})$, con confidence interval al $95 \%(95 \% \mathrm{CI})$ e $p$-value (p) ove riportati. Quando necessario, antipsicotici di prima generazione (first generation antipsychotics, FGA) ed antipsicotici di seconda generazione (second generation antipsychotics, SGA) sono stati differenziati e comparati rispetto a placebo e non-uso degli stessi.

\section{Schizofrenia e disturbo bipolare}

Solamente 3 studi osservazionali incontravano i criteri di inclusione, di cui 2 specifici per schizofrenia ([36],[37]) ed 1 ad includere pazienti psicosi in schizofrenia e disturbo bipolare [38], mentre non sono stati rilevati articoli distinti per il solo disturbo bipolare. 


\begin{tabular}{|c|c|c|c|c|c|c|}
\hline & $\begin{array}{l}\text { Tipologia } \\
\text { degli studi } \\
\text { inclusi }\end{array}$ & $\begin{array}{l}\text { Numero dei } \\
\text { partecipanti }\end{array}$ & $\begin{array}{l}\text { Specifico } \\
\text { per } \\
>65 a a ?\end{array}$ & $\begin{array}{c}\text { Follow- } \\
\text { up }\end{array}$ & $\begin{array}{l}\text { Classe degli } \\
\text { antipsicotici }\end{array}$ & Outcome per mortalità \\
\hline $\begin{array}{l}\text { Meesters } \\
\text { et } \\
\text { al., } 2016\end{array}$ & Coorte & 157 & $\mathrm{Si}$ & 5 anni & $\mathrm{FGA}+\mathrm{SGA}$ & $\begin{array}{l}\text { HR (FGA+SGA vs non- } \\
\text { uso })=2.13(95 \% \text { CI, 0.74- } \\
6.16), p=0.16\end{array}$ \\
\hline $\begin{array}{l}\text { Osborn } \\
\text { et } \\
\text { al., } 2007\end{array}$ & $\begin{array}{l}\text { Coorte } \\
\text { storica }\end{array}$ & 346382 & No & $\begin{array}{c}\text { Non } \\
\text { definito }\end{array}$ & $\mathrm{FGA}+\mathrm{SGA}$ & 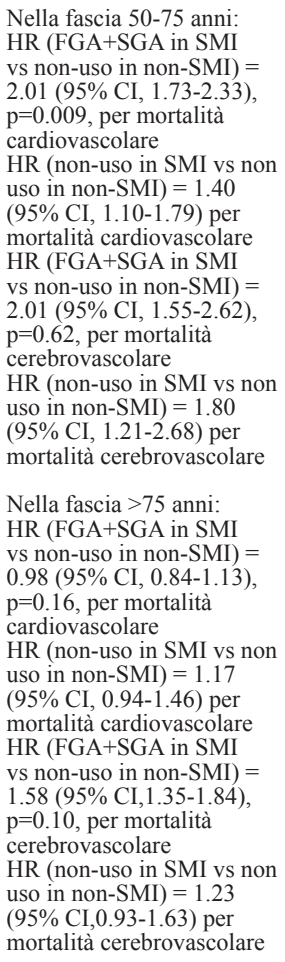 \\
\hline $\begin{array}{l}\text { Straus et } \\
\text { al., } 2004\end{array}$ & $\begin{array}{c}\text { Caso } \\
\text { controllo }\end{array}$ & 5017 & No & $\begin{array}{c}\text { Non } \\
\text { definito }\end{array}$ & $\mathrm{FGA}+\mathrm{SGA}$ & $\begin{array}{l}\text { OR (FGA+SGA vs non-uso) } \\
=3.1(95 \% \text { CI, } 1.6-6.1) \text { in } \\
>65 \text { anni vs OR (FGA+SGA } \\
\text { vs non-uso) }= \\
5.5(95 \% \text { CI, } 1.0-31.1) \text { in } \\
<65 \text { anni, per morte cardiaca } \\
\text { improvvisa } \\
\text { OR (FGA+SGA vs non-uso) } \\
=3.4(95 \% \text { CI, } 0.912 .8) \\
\text { in schizofrenia vs OR } \\
\text { (FGA+SGA vs non-uso) } \\
=3.3(95 \% \text { CI, } 1.6-6.6) \text { in } \\
\text { nonschizofrenia }\end{array}$ \\
\hline
\end{tabular}

FGA: First-Generation Antipsychotic(s), SGA: Second-Generation Antipsychotic(s), HR: Hazard Ratio, OR: Odds Ratio, CI: Intervallo di Confidenza, SMI: Severe Mental Illness, include psicosi schizofreniche e bipolari

Tabella n.1. Schizofrenia/Disturbo bipolare: studi osservazionali. 
Un recente studio osservazionale di una piccola e distinta coorte di 157 pazienti anziani di età media pari a 68 anni ed affetti da schizofrenia o disturbo schizo-affettivo, ha osservato come la prescrizione di antipsicotici di prima e seconda generazione non sia associata in maniera statisticamente significativa $(\mathrm{p}=0.16)$ con un incremento della mortalità a 5 anni per qualsiasi causa, con un HR (FGA+SGA vs non-uso) $=2.13$ (95\% CI, 0.74-6.16) [36] Uno studio caso-controllo su una popolazione di 5.017 soggetti di età mista, senza differenziare per tipo di antipsicotici usati, ma specifico per l'evento di morte cardiaca improvvisa, ha riportato un OR (FGA+SGA vs non-uso) $=3.1(95 \% \mathrm{CI}, 1.6-6.1)$ negli ultrasessantacinquenni, a fronte però di un maggiore OR $($ FGA + SGA vs non-uso $)=5.5(95 \%$ CI, 1.0-31.1) in pazienti più giovani, mentre l'OR $(\mathrm{FGA}+\mathrm{SGA}$ vs non-uso $)=3.4(95 \% \mathrm{CI}, 0.9-12.8)$ in schizofrenia in contrasto con un OR (FGA+SGA vs non-uso) $=3.3(95 \%$ CI, 1.6-6.6) in pazienti con diagnosi altra da schizofrenia [37]. Infine, uno studio a coorte storica su un ampio campione di 346.382 individui di diverse età, ha prodotto risultati validi su una popolazione affetta da psicosi sia dello spettro schizofrenico e bipolare: nella fascia di età pari a 50-75 anni, per la sola mortalità cardiovascolare, lo HR (FGA+SGA in SMI vs non-uso in non$\mathrm{SMI})=2.01$ (95\% CI, 1.73-2.33), $\mathrm{p}=0.009$, mentre lo HR (non-uso in SMI vs non uso in non-SMI) $=1.40(95 \% \mathrm{CI}, 1.10-1.79)$, invece per la mortalità cerebrovascolare, lo HR (FGA+SGA in SMI vs non-uso in non-SMI) $=2.01$ (95\% CI, 1.55-2.62), $\mathrm{p}=0.62$, a fronte di un HR (non-uso in SMI vs non uso in non-SMI) $=1.80(95 \% \mathrm{CI}, 1.21-2.68)$; nella fascia al di sopra dei 75 anni, ancora per la mortalità cardiovascolare, lo HR (FGA+SGA in SMI vs nonuso in non-SMI $)=0.98(95 \% \mathrm{CI}, 0.84-1.13), \mathrm{p}=0.16$, in contrasto con un HR (non-uso in SMI vs non uso in non-SMI) $=1.17$ (95\% CI, 0.94-1.46), e per la mortalità cerebrovascolare, lo $\mathrm{HR}$ (FGA+SGA in SMI vs non-uso in non$\mathrm{SMI})=1.58(95 \% \mathrm{CI}, 1.35-1.84), \mathrm{p}=0.10$, laddove lo HR (non-uso in SMI vs non uso in non-SMI) $=1.23(95 \% \mathrm{CI}, 0.93-1.63)$ [38].

\section{Demenze}

La letteratura riguardante la valutazione del rischio di mortalità associato ad antipsicotici in pazienti anziani con demenza è ampia: 6 revisioni sistematiche/ meta-analisi ([29],[39]-[43]) e 17 studi osservazionali ([44]-[60]) sono stati inclusi, per un totale di 20 articoli. In questa sezione, riportiamo gli studi più significativi, rimandando il lettore alla Tabella $\mathrm{n}$. 2 per una esposizione completa dei dati. 


\begin{tabular}{|c|c|c|c|c|c|c|}
\hline \multicolumn{7}{|c|}{ Revisioni sistematiche/meta-analisi } \\
\hline & $\begin{array}{l}\text { Tipologia } \\
\text { degli studi } \\
\text { inclusi }\end{array}$ & $\begin{array}{l}\text { Numero degli } \\
\text { studi/dei } \\
\text { pazienti } \\
\text { inclusi }\end{array}$ & $\begin{array}{l}\text { Specifico } \\
\text { per } \\
>65 a a ?\end{array}$ & Follow-up & $\begin{array}{l}\text { Classe degli } \\
\text { antipsicotici }\end{array}$ & Outcome per mortalità \\
\hline $\begin{array}{l}\text { Pan et al., } \\
2014\end{array}$ & RCTs & $5 / 407$ & $\mathrm{Si}$ & 1-12 mesi & $\mathrm{FGA}+\mathrm{SGA}$ & $\begin{array}{l}\mathrm{RR}(\mathrm{FGA}+\mathrm{SGA} \\
\text { discontinuation vs } \\
\text { placebo) }=0.83(95 \% \mathrm{CI} \text {, } \\
0.49-1.39), \mathrm{p}=0.48\end{array}$ \\
\hline $\begin{array}{l}\text { Pan et al., } \\
2012\end{array}$ & RCTs & 2/344 e 165 & $\mathrm{Si}$ & $\begin{array}{c}13 \\
\text { settimane } \\
\text { e } 12 \text { mesi }\end{array}$ & $\begin{array}{l}\text { FGA } \\
\text { SGA }\end{array}$ & $\begin{array}{l}\text { OR (aloperidolo vs placebo) } \\
=1.68(95 \% \mathrm{CI}, 0.72-3.92) \\
\text { HR (risperidone vs placebo) } \\
=0.58(95 \% \mathrm{CI}, 0.36-0.92), \\
\mathrm{p}=0.03\end{array}$ \\
\hline $\begin{array}{l}\text { Katz et al., } \\
2007\end{array}$ & RCTs & $4 / 895$ & $\mathrm{Si}$ & $\begin{array}{c}12 \\
\text { settimane }\end{array}$ & SGA & $\begin{array}{l}\text { HR (risperidone vs placebo) } \\
=1.26(95 \% \mathrm{CI}, 0.53-2.99)\end{array}$ \\
\hline $\begin{array}{l}\text { Haupt et } \\
\text { al., } 2006\end{array}$ & RCTs & $6 / 1721$ & $\mathrm{Si}$ & $\begin{array}{c}4-12 \\
\text { settimane }\end{array}$ & SGA & $\begin{array}{l}\text { RR (risperidone vs placebo) } \\
=1.21(95 \% \text { CI, 0.71-2.06) }\end{array}$ \\
\hline $\begin{array}{l}\text { Kryzhanovs } \\
\text { kaya et al., } \\
2006\end{array}$ & RCTs & $5 / 1662$ & $\mathrm{Si}$ & $\begin{array}{l}\text { Non } \\
\text { definito }\end{array}$ & SGA & $\begin{array}{l}\text { RD\% (olanzapina - placebo }) \\
=2.0 \%(95 \% \text { CI, n/a })\end{array}$ \\
\hline $\begin{array}{l}\text { Schneider } \\
\text { et al., } 2005\end{array}$ & RCTs & $15 / 5204$ & $\mathrm{Si}$ & $\begin{array}{c}\text { 6-26 } \\
\text { settimane }\end{array}$ & SGA & $\begin{array}{l}\text { OR }(\text { SGA vs placebo })=1.54 \\
(95 \% \text { CI, } 1.06-2.23), \mathrm{p}=0.02 \\
\text { OR (risperidone vs placebo) } \\
=1.30(95 \% \text { CI, } 0.76-2.23)\end{array}$ \\
\hline \multicolumn{7}{|c|}{ Studi osservazionali } \\
\hline & $\begin{array}{l}\text { Tipologia } \\
\text { dello } \\
\text { studio }\end{array}$ & $\begin{array}{l}\text { Numero dei } \\
\text { partecipanti }\end{array}$ & $\begin{array}{l}\text { Specifico } \\
\text { per } \\
>65 \mathbf{a a} ?\end{array}$ & Follow-up & $\begin{array}{l}\text { Classe degli } \\
\text { antipsicotici }\end{array}$ & Outcome per mortalità \\
\hline $\begin{array}{l}\text { Maust et } \\
\text { al., } 2015\end{array}$ & $\begin{array}{l}\text { Caso } \\
\text { controllo }\end{array}$ & 90786 & $\mathrm{Si}$ & $\begin{array}{l}180 \\
\text { giorni }\end{array}$ & SGA & $\begin{array}{l}\text { RD } \% \text { (aloperidolo - non } \\
\text { uso })=3.8(95 \% \mathrm{CI}, 1.0 \\
6.6), \mathrm{NNH}=26 \\
\text { RD } \% \text { (risperidone - non- } \\
\text { uso })=3.7(95 \% \mathrm{CI}, 2.2- \\
5.3), \mathrm{NNH}=27 \\
\text { RD } \% \text { (olanzapina - non-uso) } \\
=2.5(95 \% \text { CI, } 0.3-4.7), \\
\mathrm{NNH}=40 \\
\text { RD } \% \text { (quetiapina - non-uso) } \\
=2.0(95 \% \text { CI, } 0.7-3.3), \\
\mathrm{NNH}=50\end{array}$ \\
\hline $\begin{array}{l}\text { Jennum et } \\
\text { al., } 2015\end{array}$ & Coorte & 71107 & $\mathrm{Si}$ & 4 anni & SGA & $\begin{array}{l}\text { HR (FGA vs non-uso) in } \\
\text { demenza }=1.183(\mathrm{SD}, \\
0.074) \\
\text { HR (FGA vs non-uso) in } \\
\text { nondemenza }=2.026 \text { (SD, } \\
0.114) \\
\text { HR (SGA vs non-uso) in } \\
\text { demenza }=1.380(\mathrm{SD}, \\
0.042) \\
\text { HR (SGA vs non-uso) in } \\
\text { nondemenza }=1.785(\mathrm{SD}, \\
0.088)\end{array}$ \\
\hline $\begin{array}{l}\text { Sultana et } \\
\text { al., } 2014\end{array}$ & $\begin{array}{l}\text { Coorte } \\
\text { storica }\end{array}$ & 1531 & $\mathrm{Si}$ & 5 anni & SGA & $\begin{array}{l}\text { HR }(\text { SGA vs non-uso })= \\
1.05(95 \% \text { CI, } 0.87-1.26) \text { in } \\
\text { demenza vascolare }\end{array}$ \\
\hline $\begin{array}{l}\text { Piersanti et } \\
\text { al., } 2014\end{array}$ & Coorte & 696 & $\mathrm{Si}$ & 3 anni & SGA & $\begin{array}{l}\text { HR }(\text { SGA vs non-uso })= \\
2.354(95 \% \text { CI, } 1.704-3.279)\end{array}$ \\
\hline
\end{tabular}




\begin{tabular}{|c|c|c|c|c|c|c|}
\hline \multicolumn{7}{|c|}{ Studi osservazionali } \\
\hline & $\begin{array}{l}\text { Tipologia } \\
\text { dello } \\
\text { studio }\end{array}$ & $\begin{array}{l}\text { Numero dei } \\
\text { partecipanti }\end{array}$ & $\begin{array}{l}\text { Specifico } \\
\text { per } \\
>\mathbf{6 5 a} ?\end{array}$ & Follow-up & $\begin{array}{l}\text { Classe degli } \\
\text { antipsicotici }\end{array}$ & Outcome per mortalità \\
\hline $\begin{array}{l}\text { Lopez et } \\
\text { al., } 2013\end{array}$ & Coorte & 957 & $\mathrm{Si}$ & $\begin{array}{l}0.78-18 \\
\text { anni }\end{array}$ & $\begin{array}{l}\text { FGA } \\
\text { SGA }\end{array}$ & $\begin{array}{l}\text { HR }(\text { FGA vs non-uso })=0.83 \\
(95 \% \text { CI, 0.63- } 1.09) \\
\text { HR (SGA vs non-uso })=1.02 \\
(95 \% \text { CI, 0.69- } 1.50)\end{array}$ \\
\hline $\begin{array}{l}\text { Gardette et } \\
\text { al., } 2012\end{array}$ & Coorte & 534 & $\mathrm{Si}$ & 3.5 anni & $\mathrm{FGA}+\mathrm{SGA}$ & $\begin{array}{l}\text { HR (FGA+SGA vs non-uso) } \\
=1.93(95 \% \text { CI, } 1.15-3.25) \\
\text { HR (FGA+SGA vs non-uso) } \\
\text { corretto per gravità demenza } \\
=1.12(95 \% \text { CI, } 0.59-2.12)\end{array}$ \\
\hline $\begin{array}{l}\text { Gisev et al., } \\
2012\end{array}$ & Coorte & 2224 & $\mathrm{Si}$ & 9 anni & $\mathrm{FGA}+\mathrm{SGA}$ & $\begin{array}{l}\text { HR (FGA+SGA vs non-uso) } \\
=2.07 \text { (95\% CI, 1.73-2.47) } \\
\text { HR (FGA+SGA vs non-uso) } \\
\text { in comorbidità respiratoria = } \\
2.21(95 \% \text { CI, } 1.30-3.76)\end{array}$ \\
\hline $\begin{array}{l}\text { Musicco et } \\
\text { al., } 2011\end{array}$ & Coorte & 4369 & $\mathrm{Si}$ & 2 anni & $\begin{array}{l}\text { FGA } \\
\text { SGA }\end{array}$ & $\begin{array}{l}\text { HR }(\text { FGA vs non-uso })=3.7 \\
(95 \% \text { CI, 2.5-5.1 }) \\
\text { HR (SGA vs non-uso })=2.5 \\
(95 \% \text { CI, 02.0-3.0 })\end{array}$ \\
\hline $\begin{array}{l}\text { Chan et al., } \\
2011\end{array}$ & Coorte & 599 & $\mathrm{Si}$ & 18 mesi & $\mathrm{FGA}+\mathrm{SGA}$ & $\begin{array}{l}\text { RD } \%(\text { non-uso }-\mathrm{FGA}+\mathrm{SGA}) \\
=3.2(95 \% \mathrm{CI}, \mathrm{n} / \mathrm{a})\end{array}$ \\
\hline $\begin{array}{l}\text { Rossom et } \\
\text { al., } 2010\end{array}$ & Coorte & 9878 & $\mathrm{Si}$ & 30 giorni & FGA & $\begin{array}{l}\text { HR (aloperidolo vs non-uso) } \\
=2.2(95 \% \mathrm{CI}, 1.7-2.9) \\
\text { HR (risperidone vs non-uso) } \\
=1.2(95 \% \mathrm{CI}, 1.0-1.4) \\
\text { HR (olanzapina vs non-uso) } \\
=1.3(95 \% \mathrm{CI}, 1.0-1.7) \\
\text { HR (quetiapina vs non-uso) } \\
=0.8(95 \% \mathrm{CI}, 0.6-1.1)\end{array}$ \\
\hline $\begin{array}{l}\text { Simoni- } \\
\text { Wastilia et } \\
\text { al., } 2009\end{array}$ & Coorte & 996 & No & 6 mesi & $\mathrm{FGA}+\mathrm{SGA}$ & $\begin{array}{l}\text { HR (FGA+SGA vs non-uso) } \\
=0.77(95 \% \mathrm{CI}, 0.60-0.98)\end{array}$ \\
\hline $\begin{array}{l}\text { Gill et al., } \\
2007\end{array}$ & Coorte & $\begin{array}{c}9100 \\
\text { (non-istituz) } \\
4036 \text { (istituz) }\end{array}$ & $\mathrm{Si}$ & $\begin{array}{l}180 \\
\text { giorni }\end{array}$ & SGA & $\begin{array}{l}\text { HR }(\text { SGA vs non-uso })=1.32 \\
(95 \% \text { CI, } 1.12-1.54) \\
\text { HR (SGA vs non-uso) }=1.23 \\
(95 \% \text { CI, } 1.05-1.45)\end{array}$ \\
\hline $\begin{array}{l}\text { Kales et al., } \\
2007\end{array}$ & Coorte & 10651 & $\mathrm{Si}$ & 1 anno & FGA & $\begin{array}{l}\text { RR (non-use vs FGA) }=0.66 \\
(95 \% \text { CI, 0.53-0.82) }\end{array}$ \\
\hline $\begin{array}{l}\text { Trifîro et } \\
\text { al., } 2007\end{array}$ & $\begin{array}{l}\text { Caso } \\
\text { controllo }\end{array}$ & 4421 & $\mathrm{Si}$ & 9 anni & $\begin{array}{l}\text { FGA } \\
\text { SGA }\end{array}$ & $\begin{array}{l}\text { OR }(\text { FGA vs non-uso })=1.7 \\
(95 \% \text { CI, } 1.3-2.2) \\
\text { OR }(\text { SGA vs non-uso })=2.2 \\
(95 \% \text { CI } 1.2-3.9)\end{array}$ \\
\hline $\begin{array}{l}\text { Raivio et } \\
\text { al., } 2007\end{array}$ & $\begin{array}{l}\text { Caso } \\
\text { controllo }\end{array}$ & 254 & $\mathrm{Si}$ & 2 anni & $\begin{array}{l}\text { FGA } \\
\text { SGA }\end{array}$ & $\begin{array}{l}\text { HR }(\text { FGA vs non-uso })=0.68 \\
(95 \% \text { CI, 0.46- } 1.03) \\
\text { HR (SGA vs non-uso) }=0.49 \\
(95 \% \text { CI, } 0.24-0.99)\end{array}$ \\
\hline $\begin{array}{l}\text { Nonino et } \\
\text { al., } 2006\end{array}$ & Coorte & 2314 & $\mathrm{Si}$ & 1 anno & SGA & $\begin{array}{l}\mathrm{HR}(\text { SGA vs non-uso })= \\
1.023(95 \% \mathrm{CI}, 0.780-1.342)\end{array}$ \\
\hline $\begin{array}{l}\text { Suh \& } \\
\text { Saha, } 2005\end{array}$ & Coorte & 273 & $\mathrm{Si}$ & 1 anno & $\mathrm{FGA}+\mathrm{SGA}$ & $\begin{array}{l}\text { RR (non-uso vs FGA+SGA) } \\
=1.277(95 \% \mathrm{CI}, 1.1341 .437)\end{array}$ \\
\hline
\end{tabular}

Tabella n.2. Demenze. Revisione sistematica/meta-analisi e studi osservazionali. 
Una prima meta-analisi, già menzionata precedentemente, di 15 RCTs per un totale di 5.204 pazienti, specifica per antipsicotici atipici e con un follow$u p$ tra le 6 e 26 settimane, è risultata in un OR (SGA vs placebo) $=1.54(95 \%$ $\mathrm{CI}, 1.06-2.23$ ) con $\mathrm{p}=0.02$, mentre per il solo risperidone un OR (risperidone vs placebo $)=1.30(95 \%$ CI, 0.76-2.23) [29]. Due successive meta-analisi si sono concentrate sul solo risperidone: la prima (6 RCTs e 1.721 pazienti con follow up variabili tra le 4 e le 12 settimane), ha osservato un RR (risperidone vs placebo $)=1.21(95 \%$ CI, 0.71-2.06) [42]. La seconda (4 RCTs, 895 pazienti con un follow-up medio di 12 settimane), ha riportato un HR (risperidone vs placebo $)=1.26(95 \% \mathrm{CI}, 0.53-2.99)[41]$; in entrambi i casi l'associazione risultava però statisticamente non significativa. Similmente, una meta-analisi di 5 RCTs e 1.662 pazienti, questa volta specifica per olanzapina, ha ottenuto un $\mathrm{RD} \%$ (olanzapina - placebo) $=2.0 \%(95 \% \mathrm{CI}, \mathrm{n} / \mathrm{a})$ [43]. Una recente revisione sistematica di studi randomizzati controllati e studi osservazionali ha aggiunto due ulteriori RCTs [40]: un altro trial su risperidone su 165 pazienti ha calcolato un più basso HR (risperidone vs placebo) $=0.58(95 \% \mathrm{CI}, 0.36$ 0.92 ) per mortalità a 12 mesi [61]; l'unico trial su un antipsicotico di prima generazione, cioè aloperidolo, a 13 settimane su 344 pazienti, ha osservato un più elevato $\mathrm{OR}$ (aloperidolo vs placebo) $=1.68(95 \% \mathrm{CI}, 0.72-3.92)$ [62]. Una meta-analisi concettualmente differente di 5 RCTs e 407 pazienti ha valutato il rischio di mortalità a 1-12 mesi a seguito di interruzione del trattamento con antipsicotici di prima o seconda generazione, restituendo un RR (FGA+SGA discontinuation vs placebo) $=0.83(95 \% \mathrm{CI}, 0.49-1.39)$, tuttavia con $\mathrm{p}=0.48$ [39].

Un ampio e recente studio caso-controllo su 90.786 soggetti con follow up a 180 giorni ha osservato un $\mathrm{RD} \%$ (aloperidolo - non-uso) $=3.8$ (95\% CI, 1.0-6.6) con $\mathrm{NNH}=26$, un $\mathrm{RD} \%$ (risperidone - non-uso) $=3.7$ (95\% CI, 2.25.3) con $\mathrm{NNH}=27$, un $\mathrm{RD} \%$ (olanzapina - non-uso $)=2.5(95 \% \mathrm{CI}, 0.3-4.7)$ con $\mathrm{NNH}=40$, ed un $\mathrm{RD} \%$ (quetiapina - non-uso) $=2.0$ (95\% CI, 0.7-3.3), $\mathrm{NNH}=50$, con un effetto correlato alla dose per i soli antipsicotici atipici [44]. Risultati simili sono giunti da uno studio di coorte su 71.108 pazienti seguiti per 4 anni: per antipsicotici convenzionali un HR (FGA vs non-uso) $=1.183$ (deviazione standard, 0.074), mentre per antipsicotici di seconda generazione, HR (SGA vs non-uso) in demenza $=1.380$ (deviazione standard, 0.042); tuttavia, nello stesso studio il rischio appariva ancora maggiore nei pazienti anziani non dementi sottoposti a trattamento antipsicotico, con HR $($ FGA vs non-uso $)=2.026$ (deviazione standard, 0.114) e HR (SGA vs nonuso $)=1.785$ (deviazione standard, 0.088) [45]. Ancora, un lavoro più datato, ma sempre su di una ampia coorte di 10.651 individui seguiti per un anno, ha notato un rischio di mortalità minore in pazienti non trattati rispetto ai soggetti esposti all'uso di antipsicotici di prima generazione, con un RR (non-use vs 
$\mathrm{FGA})=0.66(95 \% \mathrm{CI}, 0.53-0.82)$. Nel panorama italiano, una indagine su di una coorte di 696 pazienti del centro-Italia valutati per 3 anni ha osservato un rischio di mortalità particolarmente elevato associato ad antipsicotici atipici, con un HR (SGA vs non-uso) = 2.354 (95\% CI, 1.704-3.279) [47], cosi come era avvenuto prima su una coorte di 4.369 milanesi seguiti per 2 anni, che dimostrava un simile HR (SGA vs non-uso) $=2.5$ (95\% CI, 02.0-3.0) per gli antipsicotici atipici, ed un più alto HR (FGA vs non-uso) $=3.7(95 \%$ CI, 2.55.1) per gli antipsicotici convenzionali [51]; invece, un precedente studio di coorte su 2.314 pazienti con follow up ad 1 anno e specifico per antipsicotici di seconda generazione non verificava alcuna associazione significativa tra gli stessi ed il rischio di mortalità, con un HR (SGA vs non-uso) $=1.023(95 \%$ CI, 0.780-1.342) [59]. Un più piccolo, ma interessante studio di coorte su 534 pazienti seguiti per una media di 3 anni e mezzo, ha nuovamente riportato una associazione significativa tra antipsicotici e mortalità, con un HR (FGA+SGA vs non-uso $)=1.93(95 \% \mathrm{CI}, 1.15-3.25)$, ma tale significatività andava a perdersi una volta che i dati venivano corretti per il grado di severità della demenza, con un HR (FGA+SGA vs non-uso) $=1.12$ (95\% CI, 0.59-2.12) [49]. Pur non concentrandosi sul rischio differenziale per diversi antipsicotici nel suo outcome primario, uno studio di coorte su 9878 pazienti ed un breve follow up di 30 giorni, ha riportato un aumento significativo del rischio di mortalità per aloperidolo, con HR (aloperidolo vs non-uso) $=2.2(95 \% \mathrm{CI}$, 1.7-2.9), mentre l'associazione diveniva non significativa per risperidone ed olanzapina, con un HR (risperidone vs non-uso) $=1.2(95 \%$ CI, 1.0-1.4) ed un HR (olanzapina vs non-uso) $=1.3(95 \% \mathrm{CI}, 1.0-1.7)$ rispettivamente, ed inferiore all'unità per quetiapina, con un HR (quetiapina vs non-uso) $=0.8$ (95\% CI, 0.6-1.1) [53].

Altri due studi hanno verificato una associazione significativa tra antipsicotici e mortalità in anziani con demenza ([50]-[57]), anche se non sono poco numerosi quelli in cui tale potenziale associazione è apparsa indifferente o comunque non significativa ([46],[55],[60]). In netto contrasto, alcuni articoli riportano addirittura un effetto protettivo conseguente all'utilizzo di antipsicotici, sia di prima che di seconda generazione ([48],[52],[54],[58]). Di particolare interesse, un piccolo studio caso-controllo su un totale di 254 soggetti, ha riportato un HR (FGA vs non-uso $)=0.68(95 \% \mathrm{CI}, 0.46-1.03)$ per antipsicotici di prima generazione, ed un HR (SGA vs non-uso) $=0.49(95 \%$ CI, 0.24-0.99) per antipsicotici tipici, rispetto ai decessi a 2 anni; gli stessi autori inoltre osservavano come, in assenza di trattamento antipsicotico, la necessità di contenzione fisica del paziente portasse al contrario a rischio quasi raddoppiato di mortalità, con un HR (contenzione vs non-uso) $=1.71$ (95\% CI, 1.04-2.83). 


\section{Delirium}

Per quanto riguarda il delirium, in totale, solo 1 revisione sistematica [63] ed 1 studio osservazionale [64] sono stati rilevati.

\begin{tabular}{|c|c|c|c|c|c|c|}
\hline \multicolumn{7}{|c|}{ Revisioni sistematiche/meta-analisi } \\
\hline & $\begin{array}{c}\text { Tipologia } \\
\text { degli studi } \\
\text { inclusi }\end{array}$ & $\begin{array}{l}\text { Numero degli } \\
\text { studi/dei } \\
\text { pazienti } \\
\text { inclusi }\end{array}$ & $\begin{array}{c}\text { Specifico } \\
\text { per } \\
>65 \mathrm{aa} ?\end{array}$ & $\begin{array}{l}\text { Follow- } \\
\text { up }\end{array}$ & $\begin{array}{l}\text { Classe degli } \\
\text { antipsicotici }\end{array}$ & Outcome per mortalità \\
\hline $\begin{array}{l}\text { Fok et } \\
\text { al., } \\
2015\end{array}$ & RCTs & $1 / 457$ & $\mathrm{Si}$ & 28 giorni & FGA & $\begin{array}{l}\text { Mortalità aloperidolo } 0.9 \% \\
\text { vs placebo } 2.6 \%, p=0.175\end{array}$ \\
\hline \multicolumn{7}{|c|}{ Studi osservazionali } \\
\hline & $\begin{array}{c}\text { Tipologia } \\
\text { dello } \\
\text { studio }\end{array}$ & $\begin{array}{l}\text { Numero dei } \\
\text { partecipanti }\end{array}$ & $\begin{array}{c}\text { Specifico } \\
\text { per } \\
>65 \mathrm{aa} ?\end{array}$ & $\begin{array}{l}\text { Follow- } \\
\text { up }\end{array}$ & $\begin{array}{l}\text { Classe degli } \\
\text { antipsicotici }\end{array}$ & Outcome per mortalità \\
\hline $\begin{array}{l}\text { Elie et } \\
\text { al., } \\
2009\end{array}$ & $\begin{array}{l}\text { Caso } \\
\text { controllo }\end{array}$ & 111 & $\mathrm{Si}$ & $\begin{array}{c}8 \\
\text { settimane }\end{array}$ & $\mathrm{FGA}+\mathrm{SGA}$ & $\begin{array}{l}\text { OR (FGA+SGA vs non-uso) } \\
=1.61(95 \% \mathrm{CI}, 0.88-2.96)\end{array}$ \\
\hline
\end{tabular}

Tabella n.3. Delirium. Revisione sistematica/meta-analisi e studi osservazionali.

La revisione sistematica [63] riporta un solo RCT [65] che abbia esaminato il fattore mortalità a 28 giorni in un campione di 457 pazienti anziani affetti da delirium, in cui è stato osservato come la somministrazione in profilassi di aloperidolo a basso dosaggio comportasse una percentuale di decessi pari a $0.9 \%$ rispetto al $2.6 \%$ dei soggetti esposti al placebo, con un $\mathrm{p}=0.175$.

Lo studio caso-controllo ha analizzato 326 pazienti anziani ammessi per delirium, di cui 111 erano stati trattati con antipsicotici (80\% aloperidolo, $10 \%$ risperidone, $10 \%$ altri antipsicotici); di questi, 23 morirono a fronte di una percentuale di decessi in generale pari a 55 in 8 settimane, con un OR $(\mathrm{FGA}+\mathrm{SGA}$ vs non-uso $)=1.61(95 \% \mathrm{CI}, 0.88-2.96)$ non significativo [64].

\section{Conclusioni}

In questo articolo, abbiamo presentato in maniera dettagliata i risultati di una revisione sistematica della letteratura riguardante l'associazione tra l'utilizzo di antipsicotici all'interno della popolazione psichiatrica anziana ed il rischio di mortalità. Oggi, il clinico impiega spesso tali farmaci in questo delicato sottogruppo di pazienti, a fronte di limitate prove di efficacia, 
allarmanti rapporti sui profili di sicurezza e, in definitiva, la carenza di chiare linee-guida. L'inevitabile invecchiamento della popolazione non potrà che rendere questa situazione sempre più comune, a riconferma dell'attualità di tale argomento.

Antipsicotici di prima e seconda generazione sono estesamente prescritti negli ultrasessantacinquenni, sia per indicazioni autorizzate (psicosi in schizofrenia e disturbo bipolare), ma soprattutto off-label (demenze, delirium, ed altre) [17]. Un punto di forza del nostro lavoro è stato il non essersi limitati all'analisi di una sola di queste patologie, ma l'aver riassunto, ripartiti in tre sezioni, i dati per questi maggiori campi di utilizzo dei farmaci antipsicotici nei pazienti psichiatrici anziani. Innanzitutto, è facile notare come la letteratura pubblicata sul tema non sia ugualmente distribuita, ma fortemente sbilanciata a favore dello studio dei pazienti con demenza: $80 \%$ degli articoli inclusi si concentra su questo sottogruppo, mentre solo un quinto ha valutato tale rischio in schizofrenia, disturbo bipolare e delirium. Nella schizofrenia, un unico studio ha constatato un'associazione significativa tra antipsicotici e morte cardiaca improvvisa; tuttavia, tale rischio è emerso in maggior misura nei pazienti più giovani ed in quelli non affetti da schizofrenia [37]. Gli altri due articoli inclusi confermano come il noto eccesso di mortalità per schizofrenia ([36],[38]) e disturbo bipolare [38] si protragga nell'età avanzata, in maniera apparentemente indipendente o quantomeno non interamente spiegata dall'uso di antipsicotici. A tal proposito, è importante riportare i risultati dello studio FIN-11, non accolto in questa revisione poiché non riportante analisi specifiche per la popolazione anziana, ma comunque comprendente un elevato numero di pazienti sopra i 65 anni; in questa indagine, l'esposizione a lungo termine (7-11 anni) a farmaci antipsicotici in una popolazione di 66.811 pazienti di età mista affetti da schizofrenia comporterebbe una sostanziale riduzione del rischio di mortalità, con un $\mathrm{HR}$ (FGA+SGA vs non-uso) $=0.81$ (95\% CI, 0.77-0.84) [66]. Purtroppo, non vi sono lavori simili per quanto concerne il disturbo bipolare; uno studio su una specifica coorte di 4.717 pazienti ultrasessantacinquenni non è stato incluso poiché volto alla sola comparazione tra antipsicotici atipici, ma rimane interessante notare come l'associazione con il numero di decessi sia maggiore per risperidone ed olanzapina rispetto a quetiapina in questo sottogruppo di pazienti affettivi [67]. Ipoteticamente, a fronte di una potenziale maggiore sensibilità agli effetti avversi degli antipsicotici nei pazienti affetti da disturbi dell'umore [68], può essere prudente considerare questa popolazione come esposta ad un rischio più elevato rispetto a quella schizofrenica, e quindi mantenere ulteriori cautele nell'impiego di questi farmaci.

La situazione è più complessa nell'ambito delle demenze: in 2 revisioni sistematiche/meta-analisi ([29],[43]) e 7 studi osservazionali ([44],[45],[47], 
$[50],[51],[56],[57])$ è stata osservata un'associazione significativa tra antipsicotici e mortalità, mentre per 3 revisioni sistematiche/meta-analisi ([39],[41],[42]) e 10 studi osservazionali ([46],[48],[49],[52]-[55],[58]-[60]) tale relazione è apparsa non statisticamente significativa, se non addirittura protettiva. Al cospetto di questi risultati, tenendo a mente i black box warnings della FDA, l'idea che gli antipsicotici possano condurre ad un incremento del numero dei decessi in pazienti con demenza parrebbe assai concreta. Ciononostante, a fronte di una reale mancanza di strategie alternative valide nel controllo dei sintomi comportamentali e psicotici della demenza [69], e dell'associazione con un rischio ancora maggiore per strumenti quali la contenzione fisica [58], è stato proposto che un uso attento, breve, a basso dosaggio degli antipsicotici potrebbe ancora ritenersi clinicamente appropriato, spostandosi verso un modello di cura "palliativo", cioè volto alla riduzione del grave distress in soggetti in cui l'aspettativa di vita è già intrinsecamente ridotta [70].

Per il delirium, solo 1 revisione sistematica [63] ed 1 studio osservazionale [64] hanno esaminato l'indice di mortalità nella popolazione geriatrica in relazione all'utilizzo di antipsicotici, specialmente aloperidolo, non osservando una associazione significativa ma anzi un possibile incremento della sopravvivenza. Questi pazienti sono già gravati da una prognosi particolarmente infausta, spesso dovuta alla compresenza di altre comorbidità oltre alla demenza [71]. In considerazione di questi elementi, è importante che un maggior numero di studi chiariscano il rapporto rischi/benefici per questi pazienti.

Certamente, sulla base di questi risultati, non è possibile rigettare l'ipotesi di un soggiacente meccanismo fisiopatologico comune alla condizione "vecchiaia", comportante un maggiore rischio di mortalità a seguito dell'esposizione a farmaci antipsicotici. Tuttavia, è innegabile che anche la patologia neuropsichiatrica di base abbia una sua influenza, sia essa positiva o negativa, su tale rapporto. In altre parole, se è vero che gli antipsicotici siano associati ad un maggior numero di decessi negli ultrasessantacinquenni, è altrettanto credibile che un paziente anziano affetto da schizofrenia si esponga ad un maggior rischio di morte a seguito della mancata assunzione della terapia, piuttosto che viceversa. Allo stesso modo, in considerazione dei potenziali eventi avversi associati all'utilizzo di antipsicotici nella popolazione geriatrica, il clinico dovrà decidere se sia il caso di considerare altre opzioni nel trattamento off-label di altre condizioni quali insonnia o disturbi d'ansia.

Naturalmente, questo studio ha delle apprezzabili limitazioni, talune generiche come per tutte le revisioni sistematiche, altre specifiche. 
Nonostante l'approccio sistematico nello screening delle pubblicazioni da includere, la vastità dell'argomento fa sì che l'algoritmo di ricerca utilizzato, pur rimanendo di ampio respiro, possa aver mancato di rilevare alcuni articoli significativi. A ciò si è cercato di porre rimedio aggiungendo una ricerca manuale della bibliografia; tuttavia non si può escludere che qualche studio importante sia sfuggito ai nostri criteri di ricerca.

In secondo luogo, specialmente a seguito del primo black box warning della FDA nel 2005, numerosi studi si sono concentrati sulla comparazione del rischio di mortalità tra antipsicotici di prima generazione rispetto a quelli di seconda generazione; questi studi non sono stati inclusi perché ritenuti al di là degli scopi del nostro lavoro, ma potenzialmente potrebbero apportare delle informazioni aggiuntive.

Similmente, studi su una popolazione geriatrica "generale" esposta ad antipsicotici, senza differenziazione tra pazienti con morbo di Parkinson, insonnia, ictus cerebri, ma anche schizofrenia, disturbo bipolare e depressivo, demenze ed altri, non sono stati inseriti in questa revisione poiché si è ritenuto che questo avrebbe condotto ad un'eccessiva variabilità del campione di studio che avrebbe poi compromesso un'efficace sintesi dei risultati e un'interpretazione coerente dei risultati.

Il già citato sbilanciamento di pubblicazioni a netto favore del gruppo di pazienti con demenza ci ha costretto ad includere per le altre patologie delle indagini condotte su una popolazione non specificamente anziana, cercando poi di estrapolarne dei dati applicabili a questo particolare sottogruppo; un processo certamente non scevro da significativi margini di errore. Oltre a questi limiti "quantitativi", esiste anche una problematica "qualitativa" degli studi, per cui metodologie contrastanti, differenti criteri d'inclusione/ esclusione, mancata correzione per fattori cruciali (comorbidità, gravità di malattia, presenza di malattia terminale), ed assunzione ingiustificata di rapporti causa/effetto rendono il quadro generale di difficile interpretazione. In questo contesto, sono necessari studi prospettici internazionali, in grado di appianare tali differenze e conseguentemente condurre a delle risposte più consistenti ed immediatamente applicabili nella pratica clinica.

La medicina moderna appare sempre più attenta alla sostenibilità economica, e talvolta epidemiologia, statistica ed economia sembrano prevalere sulla clinica, sulle cure, sui principi etici che la professione medica dovrebbe considerare non negoziabili. Quando poi dalla stessa revisione della letteratura emerge un quadro complesso e non univoco, un richiamo alla prudenza della gestione e assistenza appare ancora più opportuno, secondo il modello dello shared decision making tra medico e paziente. La corretta valutazione dei fattori di rischio individuali può aiutare a prendere decisioni condivise tra clinico e paziente verso scelte terapeutiche più efficaci, sicure, 
e soprattutto più appropriate. Tutto questo diventa possibile se si tiene conto del punto di vista del paziente anche, ma non solo, in termini di consenso informato, per il quale la qualità della vita e il benessere soggettivo possono essere più importanti di un aumento, peraltro non univoco, del rischio.

\section{Bibliografia}

[1] ISTAT Istituto nazionale di statistica. Il futuro demografico del Paese. Internet 2016 Available from: http://www.istat.it/it/files/2011/12/futurodemografico.pdf?title=Il+futuro+demografico+del+Paese+-+28/dic/2011++ Testo+integrale.pdf

[2] Walker ER, McGee RE, Druss BG. Mortality in mental disorders and global disease burden implications: a systematic review and meta-analysis. JAMA psychiatry 2015; 72(4): 334-341.

[3] Chesney E, Goodwin GM, Fazel S. Risks of all-cause and suicide mortality in mental disorders: a meta-review. World psychiatry 2014; 13(2): 153-160.

[4] Osborn D, Levy G, Nazareth I, King M. Suicide and severe mental illnesses. Cohort study within the UK general practice research database. Schizophrenia research 2008; 99(1-3): 134-138.

[5] Maj M. Physical health care in persons with severe mental illness: a public health and ethical priority. World psychiatry 2009; 8(1): 1-2.

[6] De Hert M, Correll CU, Bobes J, Cetkovich-Bakmas M, Cohen D, Asai I, Detraux J, et al. Physical illness in patients with severe mental disorders. I. Prevalence, impact of medications and disparities in health care. World psychiatry 2011; 10(1): 52-77.

[7] Correll CU, Detraux J, De Lepeleire J, De Hert M. Effects of antipsychotics, antidepressants and mood stabilizers on risk for physical diseases in people with schizophrenia, depression and bipolar disorder. World psychiatry 2015; 14(2): 119-136.

[8] Ray WA, Chung CP, Murray KT, Hall K, Stein CM. Atypical antipsychotic drugs and the risk of sudden cardiac death. The New England Journal of Medicine 2009; 360(3): 225-235.

[9] Danielsson B, Collin J, Jonasdottir Bergman G, Borg N, Salmi P, Fastbom J. Antidepressants and antipsychotics classified with Torsades de Pointes arrhythmia risk and mortality in older adults - a Swedish nationwide study. British journal of clinical pharmacology 2015; 81(4): 773-783.

[10] Uchida H, Mamo DC, Mulsant BH, Pollock BG, Kapur S. Increased antipsychotic sensitivity in elderly patients: evidence and mechanisms. The Journal of clinical psychiatry 2009; 70(3): 397-405. 
[11] Kleijer BC, Koek HL, van Marum RJ, Jansen PAF, Egberts TCG, Heerdink ER. Risk of acute coronary syndrome in elderly users of antipsychotic drugs: a nested case-control study. Heart 2012; 98(15): 1166-1171.

[12] Ćurković M, Dodig-Ćurković K, Erić AP, Kralik K, Pivac N. Psychotropic medications in older adults: a review. Psychiatria Danubina 2016; 28(1): 13-24.

[13] Giron MS, Forsell Y, Bernsten C, Thorslund M, Winblad B, Fastbom J. Psychotropic drug use in elderly people with and without dementia. International journal of geriatric psychiatry 2001; 16(9): 900-906.

[14] Colenda CC, Mickus MA, Marcus SC, Tanielian TL, Pincus HA. Comparison of adult and geriatric psychiatric practice patterns: findings from the American Psychiatric Association's Practice Research Network. The American journal of geriatric psychiatry 2002; 10(5): 609-617.

[15] Herzig SJ, Rothberg MB, Guess JR, Stevens JP, Marshall J, Gurwitz JH, Marcantonio ER. Antipsychotic use in hospitalized adults: rates, indications, and predictors. Journal of the American Geriatrics Society 2016; 64(2): 299305 .

[16] Trifirò G, Sini G, Sturkenboom MCJM, Vanacore N, Mazzaglia G, Caputi AP, Cricelli C, et al. Prescribing pattern of antipsychotic drugs in the Italian general population 2000-2005: a focus on elderly with dementia. International clinical psychopharmacology 2010; 25(1): 22-28.

[17] Alexopoulos GS, Streim J, Carpenter D, Docherty JP. Using antipsychotic agents in older patients. The Journal of clinical psychiatry 2004; 65 Suppl 2: 5-99.

[18] AIFA. Agenzia Italiana del Farmaco. Nota informativa sull'utilizzo di Risperidal e Belivon (risperidone) nel controllo dei sintomi comportamentali in demenza [Internet]. 2005 Available from: https://farmaci.agenziafarmaco.gov. it/aifa/servlet/PdfDownloadServlet?pdfFileName=footer_000813_038250_ RCP.pdf\&retry $=0 \&$ sys $=\mathrm{m} 0 \mathrm{~b} 113$

[19] Fountoulakis KN, Nimatoudis I, Iacovides A, Kaprinis G. Off-label indications for atypical antipsychotics: A systematic review. Annals of general hospital psychiatry 2004; 3(1): 4 .

[20] Weiss E, Hummer M, Koller D, Pharmd, Ulmer H, Fleischhacker WW. Offlabel use of antipsychotic drugs. Journal of clinical psychopharmacology 2000; 20(6): 695-698.

[21] Ownby RL. Clinical trials in geriatric disorders. Current psychiatry reports 2001; 3(1): 11-12.

[22] Gardner DM, Baldessarini RJ, Waraich P. Modern antipsychotic drugs: a critical overview. CMAJ 2005; 172(13): 1703-1711.

[23] Jeste DV, Maglione JE. Atypical antipsychotics for older adults: are they safe and effective as we once thought? Journal of comparative effectiveness research 2013; 2(4): 355-358. 
[24] Jeste DV. Tardive dyskinesia rates with atypical antipsychotics in older adults. The Journal of clinical psychiatry 2004; 65, Suppl 9: 21-24.

[25] Jin H, Meyer JM, Jeste DV. Atypical antipsychotics and glucose dysregulation: a systematic review. Schizophrenia research 2004; 71(2-3): 195-212.

[26] Jeste DV, Blazer D, Casey D, Meeks T, Salzman C, Schneider L, Tariot P, et al. ACNP White Paper: update on use of antipsychotic drugs in elderly persons with dementia. Neuropsychopharmacology 2008; 33(5): 957-970.

[27] Liperoti R, Mor V, Lapane KL, Pedone C, Gambassi G, Bernabei R. The use of atypical antipsychotics in nursing homes. The Journal of clinical psychiatry 2003; 64(9): 1106-1112.

[28] CSM Committee on Safety of Medicines. Atypical antipsychotics drugs and stroke. Internet. 2004 (cited 2016 Feb 2). Available from: http://mhra.gov.uk/ home/groups/plp/documents/drugsafetymessage/con019488.pdf

[29] Schneider LS, Dagerman KS, Insel P. Risk of death with atypical antipsychotic drug treatment for dementia: meta-analysis of randomized placebo-controlled trials. JAMA 2005; 294(15): 1934-1943.

[30] US FDA Food and Drug Administration. Public health advisory: deaths with atypical antipsychotics in elderly patients with behavioural disturbances Internet. 2005 (cited 2016 Feb 2). Available from: http://www.fda.gov/Drug/ DrugSafety/ucm053171.htm

[31] Schneeweiss S, Setoguchi S, Brookhart A, Dormuth C, Wang PS. Risk of death associated with the use of conventional versus atypical antipsychotic drugs among elderly patients. CMAJ 2007; 176(5): 627-632.

[32] Wang PS, Schneeweiss S, Avorn J, Fischer MA, Mogun H, Solomon DH, Brookhart MA. Risk of death in elderly users of conventional vs. atypical antipsychotic medications. The New England journal of medicine 2005; 353(22): 2335-2341.

[33] Nasrallah HA, White T, Nasrallah AT. Lower mortality in geriatric patients receiving risperidone and olanzapine versus haloperidol: preliminary analysis of retrospective data. The American journal of geriatric psychiatry 2004; 12(4): 437-439.

[34] US FDA. Information for healthcare professionals: information on conventional antipsychotics. Internet. 2008 (cited 2016 Feb 2). Available from: http://www.fda.gov/Drug/DrugSafety/ucm124830.htm

[35] Leon C, Gerretsen P, Uchida H, Suzuki T, Rajji T, Mamo DC. Sensitivity to antipsychotic drugs in older adults. Current psychiatry reports 2010; 12(1): 28-33.

[36] Meesters PD, Comijs HC, Smit JH, Eikelenboom P, de Haan L, Beekman ATF, Stek ML. Mortality and Its Determinants in Late-Life Schizophrenia: A 5-Year Prospective Study in a Dutch Catchment Area. The American journal of geriatric psychiatry 2016; 24(4): 272-277. 
[37] Straus SMJM, Bleumink GS, Dieleman JP, van der Lei J, Jong GW, Kingma $\mathrm{JH}$, Sturkenboom MCJM, et al. Antipsychotics and the risk of sudden cardiac death. Archives of internal medicine 2004; 164(12): 1293-1297.

[38] Osborn DPJ, Levy G, Nazareth I, Petersen I, Islam A, King MB. Relative risk of cardiovascular and cancer mortality in people with severe mental illness from the United Kingdom's General Practice Rsearch Database. Archives of general psychiatry 2007; 64(2): 242-249.

[39] Pan Y-J, Wu C-S, Gau SS-F, Chan H-Y, Banerjee S. Antipsychotic discontinuation in patients with dementia: a systematic review and metaanalysis of published randomized controlled studies. Dementia and geriatric cognitive disorders 2014; 37(3-4): 125-140.

[40] Pratt N, Roughead EE, Salter A, Ryan P. Choice of observational study design impacts on measurement of antipsychotic risks in the elderly: a systematic review. BMC medical research methodology 2012; 12(1): 72.

[41] Katz I, de Deyn P-P, Mintzer J, Greenspan A, Zhu Y, Brodaty H. The efficacy and safety of risperidone in the treatment of psychosis of Alzheimer's disease and mixed dementia: a meta-analysis of 4 placebo-controlled clinical trials. International journal of geriatric psychiatry 2007; 22(5): 475-484.

[42] Haupt M, Cruz-Jentoft A, Jeste D. Mortality in elderly dementia patients treated with risperidone. Journal of clinical psychopharmacology 2006; 26(6): 566-570.

[43] Kryzhanovskaya LA, Jeste DV, Young CA, Polzer JP, Roddy TE, Jansen $\mathrm{JF}$, Carlson JL, et al. A review of treatment-emergent adverse events during olanzapine clinical trials in elderly patients with dementia. The Journal of clinical psychiatry 2006; 67(6): 933-945.

[44] Maust DT, Kim HM, Seyfried LS, Chiang C, Kavanagh J, Schneider LS, Kales HC. Antipsychotics, other psychotropics, and the risk of death in patients with dementia: number needed to harm. JAMA psychiatry 2015; 48109: 1-8.

[45] Jennum P, Baandrup L, Ibsen R, Kjellberg J. Increased all-cause mortality with use of psychotropic medication in dementia patients and controls: A population-based register study. European neuropsychopharmacology 2015; 25(11): 1906-1913.

[46] Sultana J, Chang CK, Hayes RD, Broadbent M, Stewart R, Corbett A, Ballard C. Associations between risk of mortality and atypical antipsychotic use in vascular dementia: a clinical cohort study. International journal of geriatric psychiatry 2014; 29(12): 1249-1254.

[47] Piersanti M, Capannolo M, Turchetti M, Serroni N, De Berardis D, Evangelista $\mathrm{P}$, Costantini $\mathrm{P}$, et al. Increase in mortality rate in patients with dementia treated with atypical antipsychotics: a cohort study in outpatients in Central Italy. Rivista di psichiatria 2014; 49(1): 34-40. 
[48] Lopez OL, Becker JT, Chang Y-F, Sweet RA, Aizenstein H, Snitz B, Saxton $\mathrm{J}$, et al. The long-term effects of conventional and atypical antipsychotics in patients with probable Alzheimer's disease. The American journal of psychiatry 2013; 170(9); 1051-1058.

[49] Gardette V, Lapeyre-Mestre M, Coley N, Cantet C, Montastruc J-L, Vellas B, Andrieu S. Antipsychotic use and mortality risk in community-dwelling Alzheimer's disease patients: evidence for a role of dementia severity. Current Alzheimer research 2012; 9(9): 1106-1116.

[50] Gisev N, Hartikainen S, Chen TF, Korhonen M, Bell JS. Effect of comorbidity on the risk of death associated with antipsychotic use among communitydwelling older adults. International psychogeriatrics 2012; 24(7): 1058-1064.

[51] Musicco M, Palmer K, Russo A, Caltagirone C, Adorni F, Pettenati C, Bisanti L. Association between prescription of conventional or atypical antipsychotic drugs and mortality in older persons with Alzheimer's disease. Dementia and geriatric cognitive disorders 2011; 31(3): 218-224.

[52] Chan T-C, Luk JK-H, Shea Y-F, Lau K-H, Chan FH-W, Yu GK-K, Chu L-W. Continuous use of antipsychotics and its association with mortality and hospitalization in institutionalized Chinese older adults: an 18-month prospective cohort study. International psychogeriatrics 2011; 23(10): 16401648.

[53] Rossom RC, Rector TS, Lederle FA, Dysken MW. Are all commonly prescribed antipsychotics associated with greater mortality in elderly male veterans with dementia? Journal of the American Geriatrics Society 2010; 58(6): 1027-1034.

[54] Simoni-Wastila L, Ryder PT, Qian J, Zuckerman IH, Shaffer T, Zhao L. Association of antipsychotic use with hospital events and mortality among medicare beneficiaries residing in long-term care facilities. The American journal of geriatric psychiatry 2009; 17(5): 417-427.

[55] Gill SS, Bronskill SE, Normand S-LT, Anderson GM, Sykora K, Lam K, Bell CM, et al. Antipsychotic drug use and mortality in older adults with dementia. Annals of Internal Medicine 2007; 146(11): 775-786.

[56] Kales HC, Valenstein M, Kim HM, McCarthy JF, Ganoczy D, Cunningham F, Blow FC. Mortality risk in patients with dementia treated with antipsychotics versus other psychiatric medications. The American Journal of Psychiatry 2007; 164(10): 1568-1576.

[57] Trifirò G, Verhamme KMC, Ziere G, Caputi AP, Ch Stricker BH, Sturkenboom MCJM. All-cause mortality associated with atypical and typical antipsychotics in demented outpatients. Pharmacoepidemiology and drug safety 2007; 16(5): 538-544.

[58] Raivio MM, Laurila JV, Strandberg TE, Tilvis RS, Pitkälä KH. Neither atypical nor conventional antipsychotics increase mortality or hospital 
admissions among elderly patients with dementia: a two-year prospective study. The American journal of geriatric psychiatry 2007; 15(5): 416-424.

[59] Nonino F, De Girolamo G, Gamberini L, Goldoni CA. Survival among elderly Italian patients with dementia treated with atypical antipsychotics: observational study. Neurological sciences 2006; 27(6): 375-380.

[60] Suh G-H, Shah A. Effect of antipsychotics on mortality in elderly patients with dementia: a 1-year prospective study in a nursing home. International psychogeriatrics 2005; 17(3): 429-441.

[61] Ballard C, Hanney ML, Theodoulou M, Douglas S, McShane R, Kossakowski $\mathrm{K}$, Gill R, et al. The dementia antipsychotic withdrawal trial (DART-AD): long-term follow-up of a randomised placebo-controlled trial. The Lancet. Neurology 2009; 8(2): 151-157.

[62] De Deyn PP, Rabheru K, Rasmussen A, Bocksberger JP, Dautzenberg PL, Eriksson S, Lawlor BA. A randomized trial of risperidone, placebo, and haloperidol for behavioral symptoms of dementia. Neurology 1999; 53(5): 946-955.

[63] Fok MC, Sepehry AA, Frisch L, Sztramko R, Borger van der Burg BLS, Vochteloo AJH, Chan P. Do antipsychotics prevent postoperative delirium? A systematic review and meta-analysis. International journal of geriatric psychiatry $2015 ; 30(4): 333-344$.

[64] Elie M, Boss K, Cole MG, McCusker J, Belzile E, Ciampi A. A retrospective, exploratory, secondary analysis of the association between antipsychotic use and mortality in elderly patients with delirium. International psychogeriatrics 2009; 21(3): 588-592.

[65] Wang W, Li H-L, Wang D-X, Zhu X, Li S-L, Yao G-Q, Chen K-S, et al. Haloperidol prophylaxis decreases delirium incidence in elderly patients after noncardiac surgery: a randomized controlled trial. Critical care medicine 2012; 40(3): 731-739.

[66] Tiihonen J, Lönnqvist J, Wahlbeck K, Klaukka T, Niskanen L, Tanskanen A, Haukka J. 11-year follow-up of mortality in patients with schizophrenia: a population-based cohort study (FIN11 study). The Lancet 2009; 374: 620-627.

[67] Bhalerao S, Seyfried LS, Kim HM, Chiang C, Kavanagh J, Kales HC. Mortality risk with the use of atypical antipsychotics in later-life bipolar disorder. Journal of geriatric psychiatry and neurology 2012; 25(1): 29-36.

[68] Gao K, Kemp DE, Ganocy SJ, Gajwani P, Xia G, Calabrese JR. Antipsychoticinduced extrapyramidal side effects in bipolar disorder and schizophrenia: a systematic review. Journal of clinical psychopharmacology 2008; 28(2): 203-209.

[69] Azermai M. Dealing with behavioral and psychological symptoms of dementia: a general overview. Psychology Research and Behavior Management 2015; 8: 181. 
[70] Treloar A, Crugel M, Prasanna A, Solomons L, Fox C, Paton C, Katona C. Ethical dilemmas: should antipsychotics ever be prescribed for people with dementia? The British journal of psychiatry 2010; 197(2): 88-90.

[71] Saxena S, Lawley D. Delirium in the elderly: a clinical review. Postgraduate medical journal 2009; 85(1006): 405-413.

Per corrispondenza:

Riccardo De Giorgi

Oxford City \& North-East Oxford Adult Mental Health Team

Neill Unit, Warneford Hospital

Warneford Lane, Oxford, OX3 $7 J X$

+44 (0) 7415189489

Riccardo.DeGiorgi@oxfordhealth.nhs.uk 